\title{
Efeito da complexidade da tarefa na direção da transferência bilateral em habilidades motoras seriadas
}

\author{
Dayane M. Pinho ${ }^{1}$ \\ Guilherme M. Lage ${ }^{1,2}$ \\ Herbert Ugrinowitsch ${ }^{3}$ \\ Rodolfo N. Benda ${ }^{3}$
}

https://doi.org/10.5628/rpcd.07.02.209

\author{
${ }^{1}$ Laboratório do Comportamento Humano (LACOH) \\ Faculdades Unidas do Norte de Minas \\ Brasil \\ ${ }^{2}$ Faculdade de Ciências da Saúde (FCS) \\ Universidade FUMEC \\ Brasil \\ ${ }^{3}$ Grupo de Estudo em Desenvolvimento e \\ Aprendizagem Motora (GEDAM) \\ Universidade Federal de Minas Gerais \\ Brasil
}

\section{RESUMO}

O efeito da complexidade da tarefa na direção da transferência bilateral em habilidades motoras seriadas foi investigado através de dois experimentos. Vinte participantes praticaram uma tarefa de menor complexidade (digitação de uma seqüência de teclas) no primeiro experimento, e outros vinte participantes praticaram uma tarefa de maior complexidade (posicionamento de bolas de tênis entre recipientes) no segundo experimento. A meta para ambas as tarefas foi executar os movimentos na maior velocidade possível. Em ambos os experimentos, os sujeitos foram designados aleatoriamente para: 1) grupo de sujeitos que praticou com a mão preferida e foi testado com a mão não-preferida (GMP) e outro grupo que praticou com a mão não-preferida e foi testado com a mão preferida (GMNP). Os experimentos constaram de pré-teste, aquisição e pós-teste. As análises entre o pré e o pós-teste indicaram que, independente da direção da transferência e complexidade da tarefa, os dois grupos se beneficiaram da transferência bilateral, tanto em termos de desempenho quanto de consistência. A análise dos resultados entre o fim da aquisição e o pós-teste permitiu observar uma relação inversa entre a direção da transferência e complexidade da tarefa. Os resultados são discutidos em termos do tipo de processamento requerido nas tarefas.

Palavras-chave: aprendizagem motora, efeito de transferência, transferência bilateral, complexidade da tarefa

\begin{abstract}
Effect of task complexity in the bilateral transfer direction in serial motor skills

The effect of task complexity in the bilateral transfer direction in serial motor skills was investigated through two experiments. In the first, twenty participants performed a less complex task (typing a key sequence) and in the second, another group of twenty participants performed a more complex task (positioning). The goal in both tasks was to perform the movements as quickly as possible. In both experiments, the subjects were randomly assigned 1) to the group that practiced with the preferred hand (GPH) and was tested with the non-preferred hand or 2) to the group that practiced with the non-preferred hand (GNPH) and was tested with the preferred hand. The experiment phases consisted of pre-test, acquisition and post-test. The analysis between pre and post-test indicated that independently of the direction of bilateral transfer and task complexity, all groups had the benefits of bilateral transfer in terms of performance and consistency. The analysis between the end of acquisition and the post-test pointed out an inverse relation between the direction of transfer and task complexity. The results are discussed in terms of the kind of processing required in the tasks.
\end{abstract}

Key-words: motor learning, transfer effect, bilateral transfer, task complexity 


\section{INTRODUÇÃO}

Aprendizagem motora é o conjunto de processos associados com a prática ou a experiência, conduzindo a mudanças relativamente permanentes na capacidade de executar performance habilidosa(16).

Mudanças são observadas no eixo temporal e podem ser caracterizadas como fruto da crescente interação entre vários elementos envolvidos no desempenho motor. De acordo com Manoel(11), esses elementos correspondem aos órgãos sensoriais, à percepção, à tomada de decisão, à programação, ao sistema de feedback e ao sistema muscular. Através do fortalecimento dessa interação, ocorre uma gradativa transposição de estados desorganizados para estados organizados, emergindo assim, comportamentos que podem ser distinguidos por padrões espaço-temporais bem definidos, com metas no ambiente, caracterizados como habilidades ou tarefas motoras.

Dentre os estudos sobre habilidades motoras, um tema que tem despertado interesse dos pesquisadores é como uma habilidade aprendida com um membro pode ser transferida para o outro, o que é conhecido como transferência bilateral. Estudos sobre a transferência bilateral indicam que a aprendizagem é independente do membro efetor $(5,7,8,13)$. Em outras palavras, o desempenho de um membro pode ser otimizado pela prática do membro homólogo. De forma geral, as explanações sobre a transferência bilateral se baseiam na aprendizagem de elementos cognitivos envolvidos na execução das habilidades ${ }^{(7}$, 8) e nos mecanismos de controle motor, como programação motora, que definem os aspectos temporais e espaciais requeridos nos movimentos $(5,13)$. De acordo com Wrisberg(24), o achado mais significante sobre transferência bilateral é que essa transferência é assimétrica entre os membros, ou seja, existem diferenças em termos de direção da transferência. Em situações práticas em que a preferência manual não é restringida, observa-se uma tendência de utilização inicial do lado preferencial ${ }^{1}$, para posteriormente praticar com o lado não preferencial. Este comportamento pode estar relacionado à maior segurança e conforto gerados na execução com o membro preferido. Entretanto, resultados de pesquisa são contraditórios em relação a melhor direção da transferência. Resultados de pesquisas têm demonstrado que a melhor direção da transferência pode ocorrer do membro preferido para o não-preferido $(2,6,23)$ ou do membro não-preferido para o preferido $(9,10,15,17) \mathrm{e}$ ainda, que a direção da transferência entre membros depende de parâmetros específicos da tarefa, como força e $\operatorname{timing}(18,19,20)$. Como exemplo, Thut, Cook, Regard, Leenders, Haslband e Landis(20) utilizaram uma tarefa de desenho de padrões geométricos e observaram que, para a medida de tempo de movimento, a direção da transferência mais efetiva foi do membro preferencial para o membro não-preferencial, enquanto para a medida de precisão espacial a melhor direção ocorreu no sentido contrário.

Diferentes hipóteses explanativas são encontradas sobre os níveis de ativação hemisférica gerados pelo membro praticado, preferencial ou não-preferencial, e pela característica das tarefas. Taylor e Heilman(17) afirmam que a prática com a mão não-preferencial leva a uma ativação cerebral bihemisférica, facilitando assim a transferência para o membro preferencial. Por outro lado, a prática com a mão preferencial levaria a uma maior ativação do hemisfério esquerdo levando a um menor grau de transferência para o membro não-preferencial. Já para Teixeira ${ }^{(19)}$, determinados componentes da tarefa como o controle de força e timing coincidente são controlados com a mesma proficiência por ambos os hemisférios cerebrais, o que gera uma transferência simétrica entre os membros nesses parâmetros.

Em uma análise restrita aos estudos que utilizaram tarefas de natureza seriada, a direção mais efetiva da transferência entre membros foi do membro nãopreferencial para o membro preferencial $(9,10,15,17)$. Esses resultados suportam a hipótese de Taylor e Heilman (17) sobre os diferentes níveis de processamento gerados pela prática com o membro preferencial ou não-preferencial.

Resultados de estudos recentes que utilizaram medidas obtidas por neuroimagem e eletromiografia, apontam para uma relação entre complexidade da tarefa e ativação entre os hemisférios. Tinazzi e Zanette(21) encontraram uma maior ativação bihemisférica, em movimentos mais complexos. Assim, uma maior complexidade geraria maiores interações inter-hemisféricas trazendo maiores vantagens para o sistema ${ }^{(4,14)}$. Apoiado nos achados descritos acima, pode-se esperar que em tarefas de maior complexidade, a transferência entre membros seja 
simétrica. Isto contraria a hipótese de que em movimentos complexos a melhor direção da transferência é do membro não-preferencial para o preferencial(15). Entende-se o aumento da complexidade da tarefa como o aumento do número de elementos ${ }^{(3)}$, ou mais especificamente, um maior número de graus de liberdade envolvidos na execução da habilidade $(1,22)$. Em suma, resultados de estudos que utilizaram tarefas de natureza seriada sugerem que a direção mais efetiva da transferência entre membros parte do membro não-preferencial para o membro preferencial. Entretanto, a possibilidade de diferentes níveis de ativação hemisférica emergir não só pela prática de um determinado membro(17), mas também pela complexidade da tarefa $(4,14,21)$, justifica a realização de novas pesquisas sobre a direção da transferência entre membros. Dessa forma, foi objetivo deste estudo investigar o efeito da complexidade da tarefa na direção da transferência bilateral em habilidades seriadas. Para isso, foram realizados dois experimentos com tarefas seriadas de diferentes complexidades.

\section{EXPERIMENTO 1 - TAREFA DE MENOR COMPLEXIDADE MÉTODO}

\section{Amostra}

Vinte sujeitos universitários, de ambos os sexos, na faixa etária entre 18 e 35 anos, participaram como voluntários desse experimento. Todos os sujeitos eram inexperientes na tarefa e participaram com consentimento livre e esclarecido.

\section{Instrumentos e Tarefa}

Para a determinação do índice de dominância lateral, foi empregado o Inventário de Dominância Lateral de Edimburgo(12) e uma pergunta sobre a auto-classificação do lado preferencial. A tarefa consistiu em digitar uma seqüência de teclas pré-determinadas $(2,8$, 6 e 4), com o dedo Indicador, na região alfanumérica de um teclado. A tarefa teve como meta realizar os movimentos em maior velocidade possível. Foi utilizado um software desenvolvido especificamente para o controle de tarefas seriadas no teclado do computador e o armazenamento de dados sobre o tempo total. O tempo total foi definido pelo momento entre o pressionamento da primeira tecla e o pressionamento da última tecla. Os dados foram registrados no microcomputador ao final de cada tentativa.

\section{Delineamento Experimental}

Os participantes foram designados aleatoriamente para dois grupos de prática: 1) Grupo MãoPreferencial (GMP) e 2) Grupo Mão NãoPreferencial (GMNP). O experimento constou de três fases: 1) pré-teste com cinco tentativas de prática, 2) fase de aquisição com 30 tentativas de prática com o membro contrário ao utilizado no pré-teste e, cinco minutos após a aquisição, 3) pós-teste com cinco tentativas de prática com a mão utilizada no pré-teste. A denominação GMP ou GMNP relacionase à mão que executou a tarefa na fase de aquisição. O GMP realizou o pré-teste com a mão não-preferencial, a fase de aquisição com a mão preferencial e o pós-teste com a mão não-preferencial. O tratamento inverso foi aplicado ao GMNP, ou seja, os sujeitos iniciaram com a mão preferencial, praticaram com a mão não-preferencial e foram testados novamente com a mão preferencial.

\section{Procedimentos}

Inicialmente, os sujeitos preencheram o Inventário de Dominância Lateral de Edimburgo e responderam a questão sobre auto-definição da dominância lateral. Análises indicaram o lado direito como o preferido a todos os sujeitos. Em seguida, foram fornecidas instruções padronizadas sobre a tarefa. A informação "inicie a tentativa" era fornecida na tela do microcomputador e o sujeito iniciava a seqüência de digitação. Cinco segundos após o término de cada tentativa foi fornecido aos sujeitos o conhecimento de resultados (CR) em relação ao tempo total de movimento. O CR não foi fornecido durante o préteste e o pós-teste.

\section{Tratamento dos Dados}

Os dados foram organizados em blocos de 5 tentativas em todas as fases do experimento, tanto para a medida de desempenho quanto para a medida do desvio padrão do desempenho. O teste de Kolmogorov-Smirnov foi aplicado para verificar a normalidade dos dados, o qual indicou uma distribuição normal dos mesmos. As análises inferenciais foram realizadas através de três Anovas two-way (2 grupos X 2 blocos no pré-teste e pós-teste; 2 grupos $\mathrm{X} 6$ blocos na fase de aquisição; 2 grupos X 2 blocos entre o último bloco da aquisição e o bloco do pó- 
teste). Para análises Post Hoc, foi utilizado o teste de Tukey, quando necessário. $\mathrm{O}$ valor de significância adotada foi de $\mathrm{p}<0,05$. Essas análises foram realizadas para permitir inferências sobre a transferência da aprendizagem entre membros e a melhor direção da transferência (pré e pós-teste), as mudanças no comportamento durante a prática em relação ao membro praticado (aquisição) e a direção da transferência entre membros do final da prática para o pós-teste (último bloco da aquisição e bloco do pós-teste).

\section{RESULTADOS}

\section{Análise entre o pré e o pós-teste}

A análise do desempenho entre o pré e pós-teste (Figura 1) não indicou diferença significativa entre Grupos $[F(1,18)=0,42, p=0,52]$, assim como na interação Grupos X Blocos $[F(1,18)=0,12, p=0,72]$. Foi encontrada uma diferença significativa para $\mathrm{O}$ fator Blocos $[\mathrm{F}(1,18)=14,65, \mathrm{p}<0,01]$. O teste Post Hoc de Tukey indicou que o bloco do pré-teste apresentou maior tempo de resposta comparado ao bloco do pós-teste $(p<0,01)$.

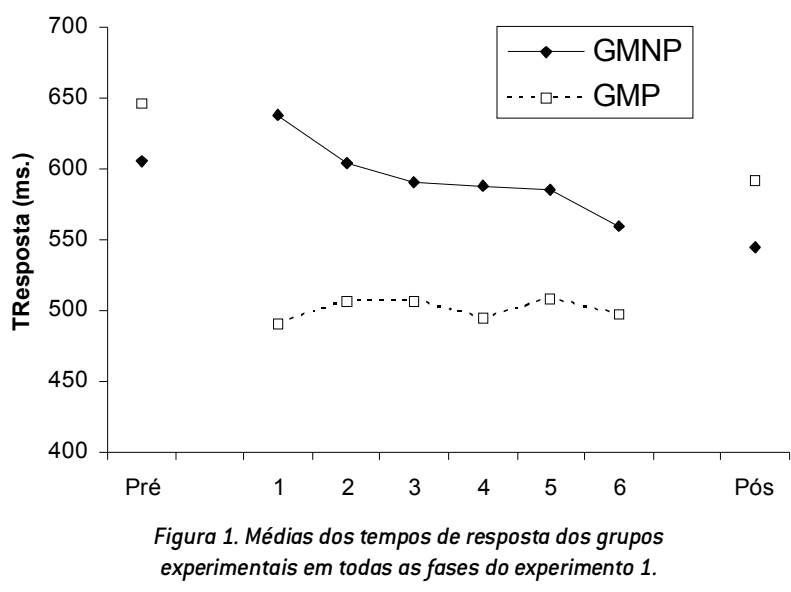

A análise do desvio padrão do desempenho não indicou diferença significativa entre Grupos $[F(1,18)=0,005, p=0,94]$, Blocos $[F(1,18)=1,66$, $\mathrm{p}=0,21]$ e na interação entre Grupos X Blocos $[\mathrm{F}(1,18)=1,98, \mathrm{p}=0,17]$.

\section{Análise da fase de aquisição}

A análise do desempenho na fase de aquisição (Figura 1) indicou diferença significante para o fator
Grupos $[\mathrm{F}(1,18)=4,57, \mathrm{p}<0,05]$, Blocos

$[F(5,90)=2,43, p<0,05]$ e na interação entre Grupos $\mathrm{X}$ Blocos $[\mathrm{F}(5,90)=2,91, \mathrm{p}<0,01]$. O teste Post Hoc de Tukey conduzido no fator Grupos indicou que o GMP apresentou menor tempo de resposta comparado ao GMNP $(p<0,05)$. Na análise do fator Blocos, o 1 o bloco da fase de aquisição apresentou maior tempo de resposta comparado ao $6^{\circ}$ bloco $(p<0,05)$. A análise da interação entre Grupos X Blocos mostrou que o $1^{\circ}$ bloco do GMNP apresentou maior tempo de resposta comparado ao $1^{\circ}$ bloco do GMP $(\mathrm{p}<0,01)$.

$\mathrm{Na}$ análise do desvio padrão do desempenho da fase de aquisição (Figura 2) não houve diferença entre Grupos $[\mathrm{F}(1,18)=0,16, \mathrm{p}=0,68]$, Blocos $[F(5,90)=1,21, p=0,30]$ e na interação entre Grupos $\mathrm{X}$ Blocos $[\mathrm{F}(5,90)=1,02, \mathrm{p}=0,4]$.

\section{Análise entre o último bloco da aquisição e o bloco do pós-teste}

A análise de desempenho (Figura 1) não indicou diferença significante para o fator Grupos $[F(1,18)=0,02, p=0,88]$. Entretanto, foi detectada diferença para o fator Blocos $[F(1,18)=8,88$, $\mathrm{p}<0,01]$ e na interação entre Grupos X Blocos $[\mathrm{F}(1,18)=17,23, \mathrm{p}<0,01]$. O teste Post Hoc de Tukey conduzido entre os blocos indicou que o último bloco da aquisição apresentou menor tempo de resposta comparado ao bloco do pós-teste $(\mathrm{p}<0,01)$. Na análise da interação entre Grupos X Blocos, o último bloco da aquisição do GMNP apresentou maior tempo de resposta comparado ao último bloco da aquisição do GMP $(\mathrm{p}<0,01)$. O último bloco da aquisição do GMP apresentou menor tempo de resposta comparado ao bloco do pós-teste do mesmo grupo $(\mathrm{p}<0,01)$.

A análise do desvio padrão do desempenho (Figura

2) não indicou diferença significante para o fator Grupos $[\mathrm{F}(1,18)=0,11, \mathrm{p}=0,74]$, assim como na interação entre Grupos X Blocos $[\mathrm{F}(1,18)=3,26$, $p=0,08]$. Foi encontrada diferença significante para o fator Blocos $[F(1,18)=4,37, p=0,05]$. O último bloco da aquisição apresentou menor variabilidade comparado ao bloco do pós-teste $(\mathrm{p}<0,05)$.

\section{DISCUSSÃo}

O objetivo do presente experimento foi investigar a direção da transferência bilateral em uma tarefa 
seriada considerada de menor complexidade. Os resultados indicaram que houve efeito de transferência bilateral para ambos os grupos de prática. Tanto o GMP quanto o GMNP apresentaram uma melhora do desempenho do pré-teste para o pós-teste, o que corrobora a proposição de que transferência bilateral indica que a aprendizagem é independente do membro efetor $(4,7,8,13)$. Em relação à variabilidade do desempenho, ambos os grupos apresentaram maior consistência ao final da prática comparado ao bloco do pós-teste.

Entretanto, em relação à direção da transferência, houve uma deterioração do desempenho por parte do GMP do último bloco da aquisição para o bloco do teste, o mesmo não ocorrendo para o GMNP. Este resultado sugere uma melhor transferência da mão não-preferencial para a mão preferencial em uma tarefa de menor complexidade. Este resultado corrobora prévios achados sobre a direção da transferência mais eficaz em tarefas seriadas $(9,10,15,17)$.

\section{EXPERIMENTO 2 - TAREFA DE MAIOR COMPLEXIDADE MÉTODO \\ Amostra}

Vinte sujeitos universitários, de ambos os sexos, na faixa etária entre 18 e 35 anos, participaram como voluntários deste experimento. Todos os sujeitos eram inexperientes na tarefa, não foram voluntários do experimento anterior, e participaram com consentimento livre e esclarecido.

\section{Instrumentos e Tarefa}

Para a determinação do índice de dominância lateral foi empregado o Inventário de Dominância Lateral de Edimburgo(12) e uma pergunta sobre a auto-classificação do lado dominante. A tarefa consistiu em soltar uma chave pressionada e transportar duas bolas de tênis em uma sequiência pré-definida entre quatro recipientes numerados de uma caixa de madeira (comprimento: $1 \mathrm{~m}$; largura: 0,66m; Altura: $0,10 \mathrm{~m}$ ). A tarefa teve como meta realizar os movimentos de posicionamento em maior velocidade possível. Foi utilizado um software desenvolvido especificamente para o controle de tempo e armazenamento de dados sobre o tempo total. O instrumento consistiu de um conjunto de diodos emissores de luz, uma chave de resposta para controlar o início da tarefa e uma segunda chave de resposta para marcar o final da mesma. O tempo total foi definido a partir da soltura da primeira chave e o pressionamento da segunda chave. Os dados foram registrados em um microcomputador ao final de cada tentativa.

\section{Delineamento Experimental}

O delineamento experimental foi o mesmo do primeiro experimento.

\section{Procedimentos}

Inicialmente, os sujeitos preencheram o Inventário de Dominância Lateral de Edimburgo e responderam a questão sobre auto-definição da dominância lateral, os quais indicaram o lado direito como o preferido a todos os sujeitos. Em seguida, foram fornecidas instruções padronizadas sobre a tarefa. Ao sinal "prepara", fornecido pelo experimentador, o sujeito pressionava a chave de resposta e, após um estímulo visual (acendimento dos diodos), a chave deveria ser solta para iniciar o transporte das bolas de tênis, uma a uma, com a mão pré-determinada, na seqüência pré-definida no menor tempo possível. Ao repousar a segunda bola sobre o último recipiente, uma segunda chave era acionada medindo o tempo total de movimento e caracterizando o fim da tarefa. Cinco segundos após o término de cada tentativa foi fornecido aos sujeitos o conhecimento de resultados (CR) em relação ao tempo total de movimento. O $\mathrm{CR}$ não foi fornecido no pré-teste e no pós-teste.

\section{Tratamento dos Dados}

O tratamento de dados foi o mesmo ao realizado no experimento 1. O teste de Kolmogorov-Smirnov indicou uma distribuição normal dos dados.

\section{RESULTADOS}

\section{Análise entre o pré e o pós-teste}

A análise do desempenho entre o pré e pós-teste (Figura 3) não indicou diferença significativa entre Grupos $[\mathrm{F}(1,18)=0,39, \mathrm{p}=0,53]$, assim como na interação entre Grupos X Blocos $[\mathrm{F}(1,18)=0,42$, $\mathrm{p}=0,72]$. Foi encontrada uma diferença significativa para o fator Blocos $[\mathrm{F}(1,18)=13,36, \mathrm{p}<0,01]$. O teste Post Hoc de Tukey indicou que o bloco do préteste apresentou maior tempo de resposta comparado ao bloco do pós-teste $(p<0,01)$. 


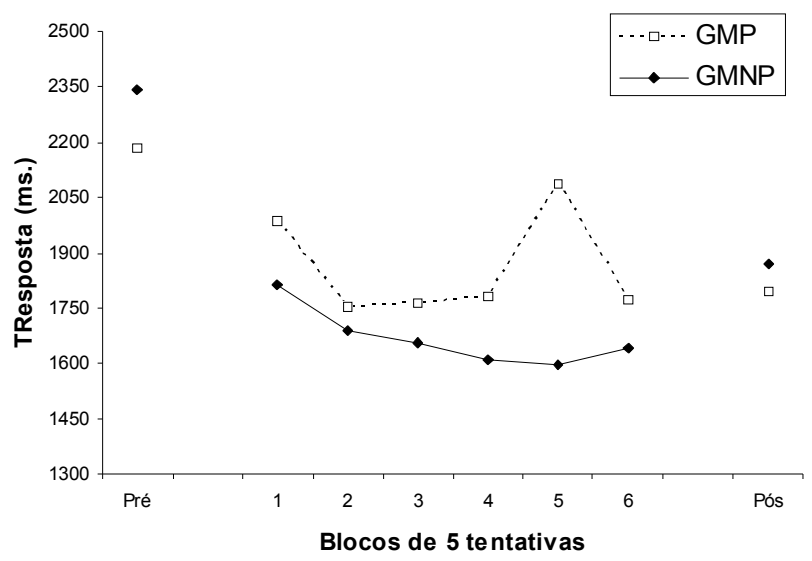

Figura 3. Médias dos tempos de resposta dos grupos experimentais em todas as fases do experimento 2 .

A análise do desvio padrão do desempenho (Figura 4) não indicou diferença significativa entre Grupos $[F(1,18)=0,005, p=0,94]$, assim como na interação entre Grupos X Blocos $[F(1,18)=0,05, p=0,81]$. Foi encontrada uma diferença significativa para o fator Blocos $[\mathrm{F}(1,18)=8,17, \mathrm{p}<0,01]$. O teste Post Hoc de Tukey indicou que o bloco do pré-teste apresentou maior variabilidade comparado ao bloco do pós-teste $(p<0,01)$.

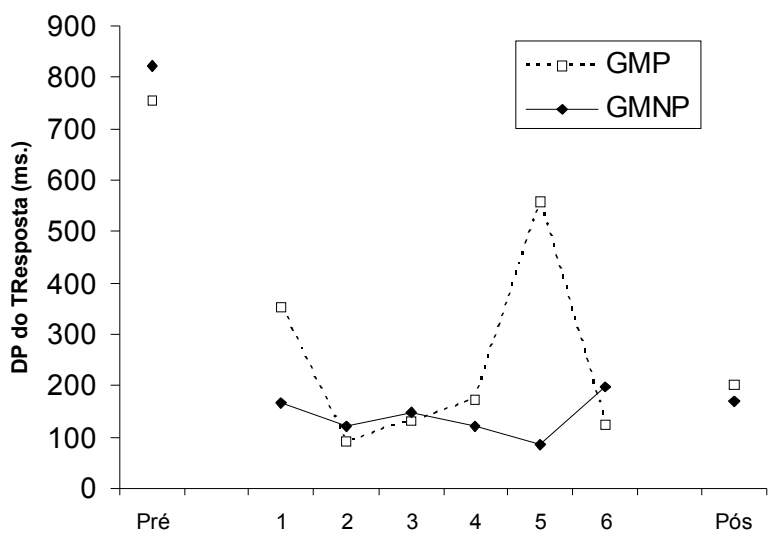

Figura 4. Desvio padrão das médias dos tempos de resposta dos grupos experimentais em todas as fases do experimento 2.

\section{Análise da fase de aquisição}

A análise do desempenho da fase de aquisição (Figura 3) indicou diferença significante para o fator Grupos $[\mathrm{F}(1,18)=4,59, \mathrm{p}<0,05]$, Blocos
$[\mathrm{F}(5,90)=4,70, \mathrm{p}<0,01]$ e na interação entre Grupos $\mathrm{X}$ Blocos $[\mathrm{F}(5,90)=3,72, \mathrm{p}<0,01]$. O teste Post Hoc de Tukey indicou que o GMNP apresentou menor tempo de resposta comparado ao GMP $(p<0,05)$. Na análise do fator Blocos, o 10 bloco da fase de aquisição apresentou maior tempo de resposta comparado ao $2^{\circ}(\mathrm{p}<0,05), 3^{\circ}, 4^{\circ}$, e $6^{\circ}$ blocos $(\mathrm{p}<0,01$, respectivamente). A análise da interação entre Grupos X Blocos mostrou que o 5o bloco do grupo GMP apresentou maior tempo de resposta que o $2^{\circ}, 3^{\circ}, 4^{\circ}$ e $6^{\circ}$ blocos $(\mathrm{p}<0,01)$.

A análise do desvio padrão do desempenho (Figura 4) indicou diferença significante para o fator Grupos $[F(1,18)=0,53, p<0,05]$ e na interação entre Grupos $\mathrm{X}$ Blocos $[\mathrm{F}(5,90)=3,35, \mathrm{p}<0,01]$. O teste Post Hoc de Tukey indicou que o GMP apresentou maior variabilidade comparado ao GMNP $(p<0,05)$. A análise da interação entre Grupos X Blocos mostrou que o 5o bloco do GMP apresentou maior variabilidade que o $2^{\circ}, 3^{\circ}, 4^{\circ}$ e $6^{\circ}$ blocos do mesmo grupo $(\mathrm{p}<0,01)$. Não houve diferença significativa para o fator Blocos $[F(5,90)=2,19, p=0,06]$.

\section{Análise entre o último bloco da aquisição e o bloco do pós-teste}

A análise do desempenho não indicou diferença significante entre Grupos $[F(1,18)=0,05, p=0,82]$. Entretanto, foi detectada diferença para o fator Blocos $[F(1,18)=7,83, p<0,01]$ e na interação entre Grupos X Blocos $[\mathrm{F}(1,18)=5,28, \mathrm{p}<0,05]$. Na análise do fator Blocos, o teste Post Hoc de Tukey indicou que o último bloco da aquisição apresentou menor tempo de resposta comparado ao bloco do pós-teste $(\mathrm{p}<0,01)$. Na análise da interação entre Grupos X Blocos, o último bloco da aquisição do GMNP apresentou menor tempo de resposta comparado ao bloco do pós-teste do mesmo grupo $(\mathrm{p}<0,01)$. A análise do desvio padrão do desempenho não indicou diferença significante para o fator Grupos $[F(1,18)=0,11, p=0,74]$, Blocos $[F(1,18)=0,21$, $\mathrm{p}=0,64]$ e na interação entre Grupos X Blocos $[\mathrm{F}(1,18)=0,92, \mathrm{p}=0,34]$.

\section{DISCUSSÃO}

O objetivo do experimento 2 foi investigar a direção da transferência bilateral em uma tarefa seriada de maior complexidade. Os resultados indicaram que 
houve efeito de transferência bilateral para ambos os grupos de prática. Tanto o GMP quanto o GMNP apresentaram uma melhora do desempenho e de sua consistência do pré-teste para o pós-teste. $\mathrm{Na}$ fase de aquisição, o grupo que praticou com a mão não-preferencial obteve melhor desempenho e consistência comparado ao grupo que praticou com a mão preferencial. Esse resultado não esperado, pode ter sido gerado mais pelos altos níveis de variabilidade encontrados no bloco 5 da fase de aquisição do que pela maior proficiência geral em todos os blocos por parte do sujeitos do GMNP.

$\mathrm{O}$ resultado mais interessante refere-se à indicação de uma melhor transferência na direção oposta à observada na tarefa de menor complexidade do primeiro experimento. O GMNP apresentou uma deterioração do desempenho do último bloco da aquisição para o bloco do pós-teste, o mesmo não ocorrendo para o GMP. Este resultado sugere uma melhor transferência da mão preferencial para a mão nãopreferencial em uma tarefa de maior complexidade. Este resultado contraria a hipótese que a melhor direção da transferência ocorre do membro não-preferencial para o preferencial(17), principalmente em tarefas complexas ${ }^{(15)}$.

\section{DISCUSSÃO FINAL E CONCLUSÃO}

A análise conjunta dos resultados dos dois experimentos possibilita inferir que, independente do nível de complexidade das tarefas seriadas utilizadas no estudo, o efeito da transferência bilateral é observado. Esses achados corroboram os resultados de estudos que encontraram efeitos positivos da prática de um determinado membro sobre o membro contralateral $(2,5,6,7,8,13,23)$.

Apesar de não ter havido diferença no desempenho entre os grupos no pós-teste, as análises realizadas entre o final da prática e a transferência para o membro homólogo mostraram um efeito da complexidade da tarefa na direção da transferência. $\mathrm{Na}$ tarefa de menor complexidade, os sujeitos que praticaram com a mão não-preferencial mantiveram o mesmo desempenho com a mão preferencial na transferência (pós-teste). Para a tarefa de maior complexidade, a melhor direção da transferência ocorreu no sentido contrário, ou seja, os sujeitos que iniciaram a prática com a mão preferencial mantiveram, na transferência, o mesmo desempenho com o membro contralateral.

$O$ resultado encontrado na tarefa de maior complexidade contraria a hipótese de Puretz ${ }^{(15)}$, que em tarefas mais complexas, a melhor direção para a transferência entre membros ocorre na direção não-preferencial para a preferencial. Apoiado nos achados de Passarotti, Banich, Sood e Wang(14) e Tinazzi e Zanette (21), é possível inferir que uma maior complexidade leva a uma maior ativação bihemisférica, tendo o sistema se beneficiado desse processo na transferência da aprendizagem do membro preferencial para o não-preferencial. Na direção contrária, esse benefício pode não ser tão claro. Seria possível sugerir que o hemisfério esquerdo (responsável pelo controle da mão preferencial direita) não se beneficie do processamento gerado pelo hemisfério direito ao mesmo nível que o hemisfério direito (responsável pelo controle da mão não-preferencial esquerda) se beneficia do processamento mais especializado na produção de movimentos do hemisfério esquerdo. De acordo com Passarotti, Banich, Sood e Wand(14), os hemisférios cerebrais são dois diferentes processadores que podem acoplar ou desacoplar os seus processos para aumentar as funções cerebrais dependendo das demandas específicas da tarefa.

A análise do ocorrido na tarefa de menor complexidade pode ser realizada no mesmo sentido discutido acima. A prática com o membro preferencial direito pode ter levado a ativação somente do hemisfério esquerdo $(4,17)$ não otimizando assim a transferência para o membro não-preferencial. Níveis iniciais de assimetria de desempenho entre os membros podem ter sido mantidos durante todas as fases do experimento. A análise conjunta dos resultados nos dois experimentos sugere uma restrição na hipótese de Taylor e Heilman ${ }^{(17)}$ sobre os diferentes níveis de ativação hemisférica gerados pela prática de um determinado membro. Devido às evidências, o fator complexidade da tarefa pode ter também um papel decisivo na ativação bihemisféri$\mathrm{ca}^{(4)}$ em habilidades seriadas. Em suma, os presentes achados apontam para um efeito da complexidade da tarefa na direção da transferência bilateral em habilidades motoras seriadas. 


\section{NOTA}

${ }^{1}$ No presente estudo assume-se que o membro direito é o membro preferencial e o membro não-preferencial é o esquerdo. Os resultados de estudos discutidos se referem aos dados obtidos com sujeitos destros.

\section{CORRESPONDÊNCIA}

\section{Prof. Guilherme Lage}

Universidade FUMEC / FCS

Faculdade de Ciências da Saúde

Departamento de Educação Física

Rua da Paisagem, 240 - Vila da Serra

Nova Lima - Minas Gerais, Brasil

CEP 34000-000

e-mail: menezeslage@gmail.com

\section{REFERÊNCIAS}

1. Bernstein $N$ (1967). The co-ordination and regulation of movement. London: Oxford Press.

2. Byrd R, Gibson M, Gleason MH (1986). Bilateral transfer across ages 7 to 17 years. Perceptual and Motor Skills 62: 87-90.

3. Campbel DJ (1988). Task complexity: a review and analysis. Academy of Management Review 13: 40-52.

4. Hausmann M, Kirk LJ, Corballis PM (2004). Influence on task complexity on manual asymmetries. Cortex 40: 103-110.

5. Hicks RE, Gualtieri CT, Schroeder SR (1983). Cognitive and motor components of bilateral transfer. Journal of General Psychology 107: 277-281.

6. Inui N (2005). Lateralization of bilateral transfer of visuomotor information in right-handers and left-handers. Journal of Motor Behavior 37: 275-283.

7. Kohl RM, Roencker DL (1980). Bilateral transfer as function of mental imagery. Jourmal of Motor Behavior 12: 197-206.

8. Kohl RM, Roencker DL (1983). Mechanism involvement during skill imagery. Journal of Motor Behavior 15: 179-190.

9. Kumar S, Mandal M (2005). Bilateral transfer of skill in left- and right-handers. Laterality: Asymmetries of Body, Brain, and Cognition 10: 345-352.

10. Lav rysen A, Helsen WF, Tremblay L, Elliott D, Adam JJ, Feys P, Buekers MJ (2003). The control of sequential aiming movements: the influence of practice and manual asymmetries on the one-target advantage. Cortex 39: 307-325.

11. Manoel EJ (2001). O diálogo no processo de aquisição de habilidade motoras. In: Guedes MGS (ed.). Aprendizagem motora: problemas e contextos. Lisboa: FMH edições.

12. Oldfield RC (1971). The assessment and analysis of handedness: the Edinburgh inventory. Neuropsychologia 9: 97-113.

13. Park J, Shea CH (2002). Effector independence. Journal of Motor Behavior 34: 253-270.

14. Passarotti AM, Banich MT, Sood RK, Wang JM (2002). A generalized role of interhemispheric interaction under attentionally demanding conditions: evidence from the auditory and tactile modality. Neuropsychologia 40: 1082-1096.

15. Puretz SL (1983). Bilateral transfer: the effects of practice on the transfer of complex dance movement patterns. Research Quarterly for Exercise and Sport 54: 48-54.

16. Schmidt RA, Lee TD (1999). Motor control and learning: $a$ behavioral emphasis. Champaign: Human Kinetics.

17. Taylor HG, Heilman KM (1980). Left-hemisphere motor dominance in righthanders. Cortex 16: 587-603.

18. Teixeira LA (2000). Timing and force components in bilateral transfer of learning. Brain and Cognition 44: 455-469.

19. Teixeira LA (2006). Intermanual transfer of timing control between tasks holding different levels of motor complexity. Laterality 11: 43-56.

20. Thut G, Cook NG, Regard N, Leenders KL, Haslband U, Landis T (1996). Intermanual transfer of proximal and distal motor engrams in humans. Experimental Brain Research 108: 321-327.

21. Tinazzi M, Zanette G (1998). Modulation of ipsilateral motor cortex in man during unimanual finger movements of different complexities. Neuroscience Letters 224: 121-124.

22. Tu rvey MT, Fitch HL, Tuller B (1982). The Bernstein perspective I: the problems of degree of freedom and context-conditioned variability. In: Kelso JA (ed.). Human motor behavior: an introduction. Hillsdale, NJ: Lawrence Erlbaum Asociates.

23. Wang J, Sainburg RL (2004). Limitations in interlimb transfer of visuomotor rotations. Experimental Brain Research 155: 1-8.

24. Wrisberg CA (1993). Levels of performance skill. In: Singer RN, Murphey M, Tennant LK (eds.). Handbook of Research on Sport Psychology. New York: MacMillan Publishing Company. 